\title{
SURF ZONE WAVE BREAKING IDENTIFICATION USING MARINE RADAR
}

\author{
Patricio Catalán ${ }^{1,4}$, Merrick Haller ${ }^{1}$, Rob Holman $^{2}$ and William Plant ${ }^{3}$ \\ This paper presents a novel combination of synchronous, large coverage marine radar, \\ calibrated pulsed Doppler radar, and video remote sensing observations from a nearshore \\ field site. The data enable the analysis of the evolution and characteristics of the remotely \\ observed signals from surf zone waves. The combination of different remote sensors allows \\ a better discrimination between breaking and non-breaking waves, such a discrimination \\ method offers the potential of more quantitative analyses of the wave breaking roller at field \\ scales. In the present work, we concentrate on quantifying the contribution of the wave \\ breaking roller to the total microwave backscatter and optical intensity signals. Analysis of \\ the power outputs of both breaking and non-breaking events demonstrates that the backscatter \\ from the wave roller is distinguishable from relict foam and appears to be independent of \\ environmental conditions. Finally, we test a volumetric scattering model against the wave \\ breaking observations. This is a new application for this type of scattering model. The model \\ treats the wave roller as a multi-phase body where most of the scattering arises from a layer \\ of water droplets representing the wave roller. The model shows an improved agreement with \\ the data as compared to the traditional surface scattering model.
}

\section{INTRODUCTION}

Wave breaking in the surf zone is an important forcing mechanism in the generation of nearshore currents and in the driving of sediment transport. At the same time, wave breaking can exhibit significant spatial and temporal variability that needs to be accounted for in the description of nearshore processes. Wave breaking in the surf zone drives the development of the wave roller, which is the turbulent body of air and water propagating with the broken wave. The presence of the roller can be a dominant factor in the mean balances of mass and momentum and in the generation of cross-shore currents. Most of the models for the hydrodynamics of the surf zone rely on the appropriate description of wave breaking (onset, frequency of occurrence) and of the roller (length scales and lifespan), therefore measurement of these quantities is highly desirable.

Remote sensing techniques can provide large spatial and long temporal coverage with a wide range of resolutions. However, estimation of wave parameters requires a proper understanding of the imaging mechanisms, which are often complex and depend on many aspects such as the nature of the scatterers,

\footnotetext{
${ }^{1}$ School of Civil and Const. Eng., Oregon State University, 220 Owen Hall., Corvallis, OR, 97331, USA

${ }^{2}$ College of Oceanic and Atmospheric Sciences, Oregon State University, Corvallis, OR, 97331, USA

${ }^{3}$ Applied Physics Lab, University of Washington, Seattle, WA, 98105, USA

${ }^{4}$ Depto. de Obras Civiles, UTFSM, Casilla 110-V, Valparaiso, Chile
} 
viewing geometry and illumination conditions to name a few. Video remote sensing has been extensively applied to nearshore monitoring over the past two decades (see Holman and Stanley, 2007, for a review), but can be affected by poor illumination conditions. Microwave sensors have also proven capable of delivering quantitative wave data, but in general the imaging mechanisms are much more complex. Furthermore, breaking waves have been shown to generate signals that deviate from traditional models such as the composite surface model, CST (e.g. Plant, 1990), most notably at low grazing angles, but the actual

contribution and nature of the scattering mechanism from breaking waves is still a subject of active research (see Wetzel, 1990, for a review). Furthermore, microwave research has focused mostly on deep water waves with only a limited amount of nearshore observations. While the presence of breaking can hamper existing algorithms of wave parameter retrieval, its distinct characteristics may yield improved discrimination algorithms, which are also desirable for nearshore studies. In this regard, it is of interest to qualify and quantify the effect on the signals of environmental parameters such as wave height and wind, as well as the effect of production and persistence of foam due to whitecapping and breaking.

In the present work we study both qualitatively and quantitatively the remote sensing signal from surf zone waves, with special emphasis on breaking waves. Our objective is to improve our understanding of the imaging mechanisms which in turn will to the development of algorithms for the retrieval of wave parameters and improved discrimination of breaking vs. non-breaking waves and relict foam. To do this, a unique field experiment was performed and some of the data analysis is presented here. In light of the results, a new model for scattering from breaking waves is discussed.

\section{EXPERIMENT DESCRIPTION}

A nearshore field experiment was conducted over a six week period at the Field Research Facility, US Army Corp of Engineers, in Duck, NC, between April 10 and May 22, 2008. Data were collected using three remote sensing instruments; one single polarization (horizontal-horizontal transmit-receive) marine radar operating at $9.45 \mathrm{GHz}$, was mounted atop a $10 \mathrm{~m}$ tower at the north end of the FRF facility, at $x=17.37 \mathrm{~m}, y=971.38 \mathrm{~m}, z=13.83 \mathrm{~m}$ in the FRF coordinate system, where $x$ is the cross-shore axis. The shoreline was approximately at $x=90 \mathrm{~m}$. The marine radar (Si-Tex RADARpc-25.9) is an active sensor with a nominal power of $25 \mathrm{~kW}$, and utilizes a $9 \mathrm{ft}$ open array antenna rotating at $44 \mathrm{rpm}$, meaning that a given point on the surface is sampled roughly every $1.36 \mathrm{~s}(0.73 \mathrm{~Hz})$. A Pulse Repetition Frequency (PRF) of $2000 \mathrm{~Hz}$ was used along with a pulse width of 80 ns resulting in an intrinsic range resolution of $12 \mathrm{~m}$, although the system internally oversamples yielding a working range resolution of $3 \mathrm{~m}$, which is constant in range. The horizontal antenna beamwidth is $0.8^{\circ}$ and the vertical beamwidth is about $25^{\circ}$. Data acquisition was designed to average 7 received waveforms thus reducing noise while decreasing the azimuthal resolution to roughly $2^{\circ}$. Special care was taken to avoid saturation of the incoming signal, by introducing the 
maximum offset possible $(-500 \mathrm{mV})$ to the received signal before it is processed by the internal logarithmic amplifier. The recorded signal is an uncalibrated grayscale intensity index $I_{r}(\theta, r, t)(0-255)$. The acquisition system was developed by Imaging Science Research. It records the relative azimuth and time (accurate to 10 $\mathrm{ms}$ ) of each sample, which enabled geolocation and synchronization with the other sensors. The maximum recorded ground range was set to $1200 \mathrm{~m}$ and covered a swath of about $270^{\circ}$ under nominal rotations speeds. 30 minute collections were recorded at the beginning of the hour throughout the duration of the experiment.

The second sensor was RiverRad, an X-band $(9.36 \mathrm{GHz})$, dual polarization (HH and VV) coherent radar developed by the Applied Physics Lab, University of Washington (APL-UW). It was deployed on the crest of the dune at the north end of the FRF property, at $x=54.40, y=936.22$ and $z=10.24 \mathrm{~m}$ in the FRF reference system. Further system details can be found in Plant et al. (2005). To maximize the overlapping area coverage with the other two sensors, the RiverRad acquisition scheme was set to collect roughly $960 \mathrm{~m}$ in range in staring mode for two minutes, after which the antennas were mechanically rotated. A total of 9 azimuthal locations separated roughly $10^{\circ}$ were collected, resulting in the partial coverage of a circular sector of about $80^{\circ}$, spanning from a direct offshore look direction to a nearly alongshore look direction at the shoreline. Clockwise and counterclockwise rotations were alternated and the system collected data almost continuously throughout the experiment. Data was delivered as received power and converted to normalized radar cross section, $\sigma_{0}\left(\theta_{i}, r, t\right)$ during post processing.

The third remote sensing system was comprised of three of the ARGUS III optical cameras that are permanently deployed at the FRF by the Coastal Imaging Lab, College of Oceanic and Atmospheric Sciences, OSU. Further details of this system can be found in Holman and Stanley (2007). For the purpose of this experiment, a rectangular pixel array was designed spanning $x=60-600 \mathrm{~m}$ and $y=500-1000 \mathrm{~m}$ with spatial resolution of $\Delta x=2 \mathrm{~m}$ and $\Delta y=5 \mathrm{~m}$ using cameras 0,3 and 1 . Video pixel intensity data $I(x, y, t)$ were collected simultaneously with the marine radar during daylight hours (7 runs per day), at a sampling rate of $2 \mathrm{~Hz}$ over 31 minutes.

In order to minimize range and resolution cell size dependencies of the signal, the marine radar data is cross-calibrated to normalized radar cross sections (NRCS, $\sigma_{0}$ ) using as reference the calibrated data output from RiverRad from simultaneous runs. The calibration is an extension of the procedure presented in Gommenginger et al. (2000).

\section{DATA ANALYSIS}

As a first step we focus on the statistical description of the data to characterize the signal according to its source. The longer time series of video and marine radar data are best suited for this task, and the RiverRad data are later used for a more complete suite of measurements. Three synchronous marine and video runs collected during a storm are used. During this time the significant wave 


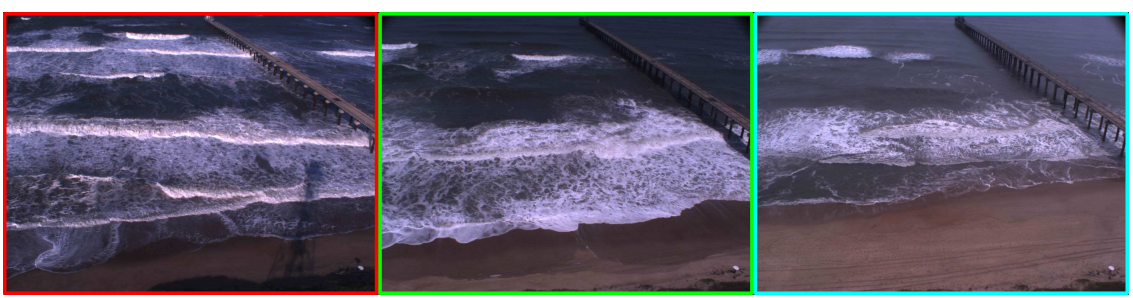

Figure 1. Video snapshots taken from Camera 1 for each of the three selected runs. From left to right, Run 9; Run 13 and Run 18

height decayed significantly over three days, while the wind decreased abruptly at first and then increased gently during the last day while changing direction from blowing onshore to offshore, as shown in Table 1. Production and persistence of remnant foam due to whitecapping and depth limited breaking also showed a decaying trend, as shown in Fig. 1.

Table 1. Summary of the wave conditions measured at the 8-m array during the storm of May 12-15, 2008 for the three selected runs. Relative (rel) directions are measured $\mathrm{CW}$ with $0^{\circ}$ pointing along the FRF $x$ axis.

\begin{tabular}{|c|c|c|c|c|c|c|c|c|}
\hline $\begin{array}{l}\text { Id. } \\
\text { No. }\end{array}$ & Date & Time & $H_{m o}$ & $T_{p}$ & $\begin{array}{c}\text { Tide } \\
\text { NAVD88 } \\
m\end{array}$ & $\begin{array}{c}\text { Wind } \\
\text { Speed } \\
\mathrm{m} / \mathrm{s}\end{array}$ & $\begin{array}{c}\text { Wind } \\
\text { Dir. } \\
{ }^{\circ} \text { TN (rel) }\end{array}$ & $\begin{array}{c}\text { Wave } \\
\text { Dir. } \\
{ }^{\circ} \text { TN (rel) }\end{array}$ \\
\hline 9 & May 13 & $22: 00$ & 3.26 & 12.5 & 0.51 & 11.6 & $56(-16)$ & $56(-16)$ \\
\hline 13 & May 14 & $18: 00$ & 1.97 & 12.5 & 0.26 & 5.4 & 229 (157) & $64(-8)$ \\
\hline 18 & May 15 & $15: 00$ & 1.47 & 11.4 & -0.44 & 7.7 & $253(181)$ & $71(-1)$ \\
\hline
\end{tabular}

Table 2. Zones definition. Values expressed in terms of the cross-shore coordinate in FRF reference system.

\begin{tabular}{|lcc|}
\hline Identifier & \multicolumn{2}{c|}{ Cross-shore Limits $(\mathrm{m})$} \\
& $x_{\min }$ & $x_{\max }$ \\
\hline Surf & 100 & 150 \\
Trough & 150 & 250 \\
Outer Bar & 250 & 350 \\
Offshore & 400 & 600 \\
\hline
\end{tabular}

Each of these data sets is rectified to the cartesian grid accounting for tidal variations, and processed on a zonal basis. The zonification is designed to further differentiate the behavior and evolution of the signals between areas of persistent, intermittent, and sporadic or no breaking. The field of view is thus divided into 4 areas which remain constant and are defined in Table 2. The aggregate of all the pixels within each zone is processed as joint and individual histograms 

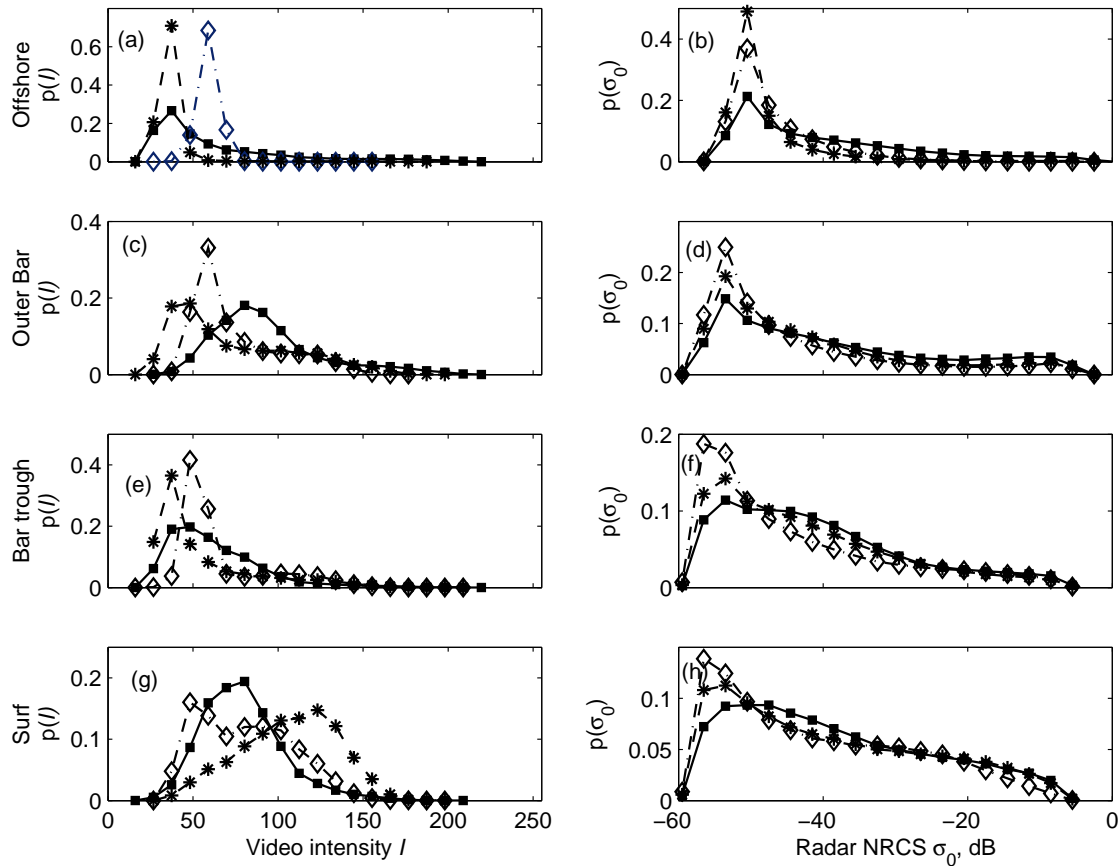

Figure 2. Video (left) and marine radar (right) histograms. Top to bottom correspond to measurements taken in the Offshore, Outer Bar, Bar trough and Surf zone boxes, respectively. Run 09 (squares, solid line); Run 13 (asterisks, dashed line); Run 18 (diamond, dash-dot line)

by counting the frequency of occurrence of a given video intensity $I$ and $\sigma_{0}$ (synchronous and collocated) pair in the ensemble of samples collected in a given zone throughout the 30-minute run. After removal of marine data below the noise level, the minimum number of usable samples was of the order of 535,000 (Camera 1, Surf zone, Run 18). The joint and individual histograms were constructed using 25 predefined bins 11 intensity values wide for video and $3 \mathrm{~dB}$ wide for the case of marine radar. Integration of the joint histogram along each axis yields the histogram of each data set. To compare between runs and zones, the histograms have been normalized by number of samples. For brevity, results presented here are from the area imaged by Camera 1. Results from the other cameras show similar behavior.

Fig. 2 shows the histograms of each sensor and zone. The video histograms show three distinct shapes. The first type is a peak at low intensity values accounting for a large fraction of the data (e.g. Run 13,Fig. 2a). This corresponds to non-breaking waves, which modulate the incident radiance on the sensor as a function of the wave slope. The dynamic range of this imaging mechanism is 
very small and spans a few bins of the histogram thus explaining the observed peakiness of the histogram. Though the shape is preserved between runs, the mean values are offset between runs owing to changes in wave conditions (e.g. height thus slope) and in camera settings such as shutter speed and aperture, which were allowed to be adjusted freely between runs depending on the illumination conditions. We ignore these differences as we are interested primarily in the shape of the histogram. A second type of shape corresponds to a peak of lesser magnitude at low video intensities followed by an exponentially decaying tail. This kind of behavior would be expected of zones where breaking takes place with some degree of foam persistency. This can be seen for instance in the offshore zone (see Fig. 2a) where Run 9 differs from the other runs due to intermittent breaking (see Fig. 1). As foam persistence becomes more pronounced, the frequency count increases at mid $I$ values, while breaking occurrence contributes at higher $I$ values. In some cases, patches of remnant foam persisting between waves and patches of dark water (non-breaking waves) may coexist resulting in bimodal histograms, for example Run 18 in the surf zone, where a clear peak at low intensities and a secondary peak at larger intensities are present. The third shape corresponds to a linear increase at low intensities with a histogram maximum at a relatively large intensity value, followed by a sharp decay at large intensities, cf. Run 13, Fig. 2g. Analysis of the pixel time series showed the occurrence of bright values at times not associated with the passage of a wave crest, thus foam patches scattering as brightly as the wave roller.

Unlike the video data, in the case of the marine radar the majority of the curves show a relatively constant shape characterized by sharp peak at low backscattered power, usually about -55 to $-50 \mathrm{~dB}$, followed by an exponential-like decay at higher power. This is consistent with probability density functions derived from a Composite Surface scattering model (e.g. Gotwols and Thompson, 1994), where the magnitude of the peak and the tail depends on ambient parameters. It is of note that the histograms follow a clear transition from low peaks and high tail values for Run 9, to high peaks and low tails for Run 18 without changing significantly the overall curve shape (e.g. Fig. 2b), especially at mid intensities. Possible reasons for this could be related to changes in the surface roughness due to wind effects or foam. For instance, the transition of Run 9 from Fig. 2 b) to h) (increasing foam) is similar to the change between runs in the trough (Fig. 2f).

Of particular interest is the effect of intermittent breaking, for instance in the outer bar (see Fig. 2d). Here the histograms are similar in shape varying only in magnitude as the wave conditions evolved. One significant aspect is the departure from the monotonic decay near $-25 \mathrm{~dB}$, where the histogram stabilizes and then increases its magnitude reaching a maximum around $-7 \mathrm{~dB}$, value after which it shows a linear decay. The increased probability values at NRCS greater than -20 $\mathrm{dB}$ can not be explained by the traditional scattering models, which would predict an exponential or exponential-like decay. It seems reasonable at this point to presume that this is related to another scattering mechanism that could be related 

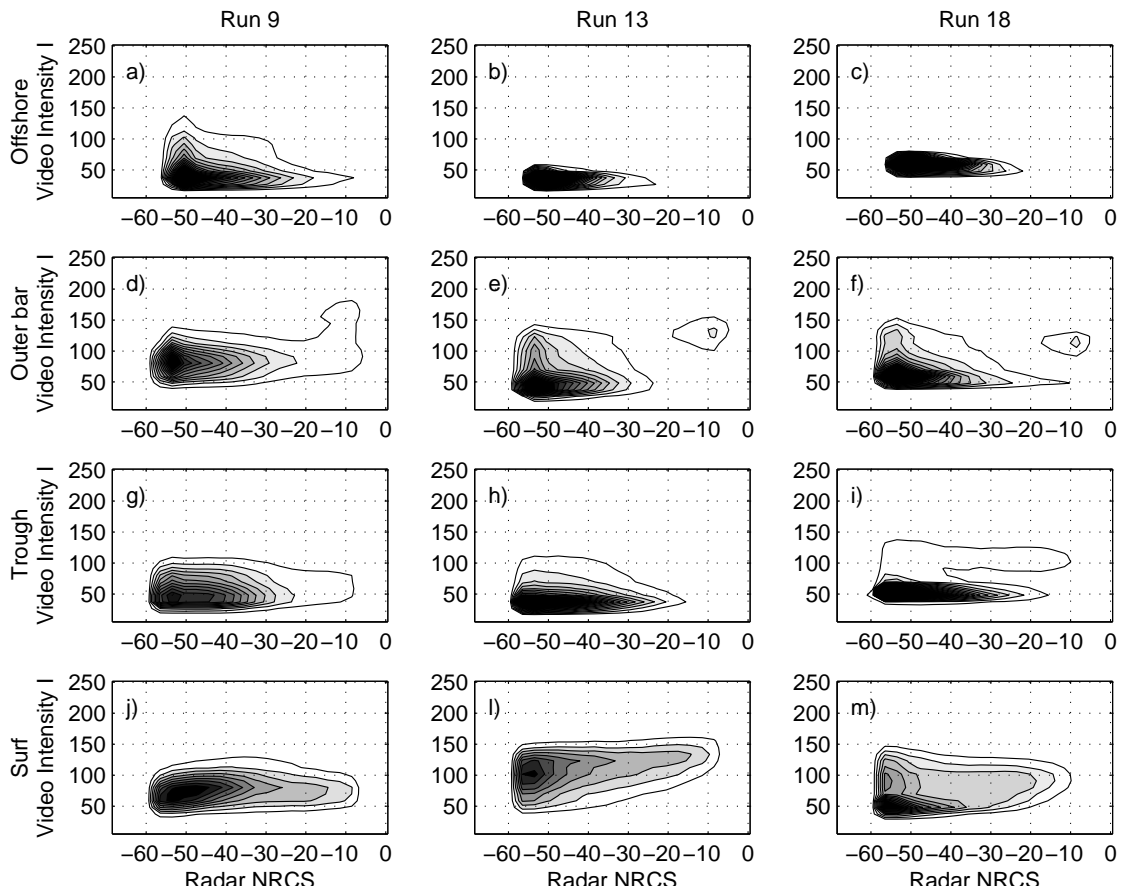

Figure 3. Joint Probability Density function for video (Camera 1) and Marine radar. Columns correspond to Runs 9, 13 and 18, respectively. Rows correspond to zones according to Table 2.

to either active breaking or the presence of foam. The linear decay at high values is artifact of the effect of waves saturating the signal and whose calibration to NRCS is linearly dependent on range and grazing angle on the $\mathrm{dB}$ scale.

The understanding of the video histogram enabled us to characterize the presence of foam but not necessarily distinguish it from active breaking. To address the effect of the source in the radar scattering characteristics per source, the joint histogram is used. Fig. 3 shows the joint histograms $(\mathrm{JH})$ for all the zones and runs considered. It can be seen that for almost all cases the peak of the $\mathrm{JH}$ occurs at relatively low video intensities and low backscattered power. This is consistent with scattering from unbroken waves over a large fraction of the wave phase. When only non-breaking waves are present (thus darker video intensities), the $\mathrm{JH}$ is concentrated over a ridge spanning a narrow range of video intensity bins but spread over a wide range of radar NRCS bins (e.g. Fig. 3b and Fig. 3c). This can be explained in terms of CST theory, where the signal is modulated by the wave slope, which induces a relatively large dynamic range for the NRCS (e.g. Plant, 1990) but narrow range for the video data (Jähne et al., 1994). Similar 
behavior can be seen in the trough for decaying wave heights (Fig. 3h and Fig. $3 i$ ), but the signal shows a slightly broader video range. A secondary ridge is also apparent in other cases (e.g. Fig. 3a, e, and f). This vertical ridge spans a wide range of video intensity bins but a relatively narrow range of backscattered power. This pattern could be explained as remnant foam inducing a large video intensity dynamic range but not producing strong microwave backscatter. Thus these two ridges can be explained by the presence of unbroken waves, slope modulations (steep waves), and to some degree foam. The presence of active breaking in turn corresponds to large $I$ and $\sigma_{0}$ or the upper right quadrant of the JH. Many of the zones exhibit a local peak in this quadrant (e.g. Fig. 3 a, d, e ,f h, and i), suggesting a clear separation between foam and active breaking for both sensors. This is consistent with intermittent breaking taking place in the offshore zone and outer bar for Run 9, when wave height was the maximum; or in the outer bar for the other two environmental conditions.

However, in the inner surf zone where wave breaking is more frequent, the JHs are not consistent with the results from the other zones. For instance, Fig. $3 \mathrm{j}$ (large wave height, strong onshore wind, large amounts of foam) do not exhibit the breaking peak, and in turn the largest video intensities correspond to mid power NRCS. On the other hand, the bulk of the signal for this case is concentrated at mid video intensities (assumed to be foam) but with radar scattering somewhat strongly from it. A similar behavior is observed for the case of low wave heights and mild wind (Fig. $3 \mathrm{~m}$ ), although in this case a peak is present at low intensities and NRCS corresponding to foam-free water. There is no discernible breaking peak and a uniform distribution across NRCS values is apparent instead. The remaining surf zone case (Fig. 31), mid wave heights and weak wind speeds, shows yet another pattern. Unlike the other two cases, there is a definite trend toward the breaking peak, although the presence of the peak itself is not so readily apparent. Part of the reason is remnant foam having intensities as bright as active breaking, and could explain the almost uniform distribution of NRCS at high video intensity values.

Thus we have characterized the scattering sources based on the information as provided by the histograms and JHs, under the assumption that the brightest optical signal corresponds to active breaking. Next, we evaluate whether these results can be used to discriminate breaking from non breaking waves on a wave-by-wave basis. The procedure is conceptually simple, in the sense that given a suitable selection of the two threshold values $I^{t}, \sigma_{0}^{t}$ it is possible to separate the breaking peak in the JH from the other sources, as defined by the following discrimination rules

$$
\begin{array}{rllll}
\text { Breaking: } & I(\vec{x}, t) & \geq I^{t} \quad \& & \sigma_{0}(\vec{x}, t) \geq \sigma_{0}^{t}, \\
\text { Foam: } & I(\vec{x}, t) & \geq I^{t} \quad \& & \sigma_{0}(\vec{x}, t)<\sigma_{0}^{t}, \\
\text { Dark Zones: } & I(\vec{x}, t) & <I^{t d}, & & \\
\text { Steep waves: } & I(\vec{x}, t) & <I^{t d} & \& & \sigma_{0}(\vec{x}, t) \geq \sigma_{0}^{t},
\end{array}
$$


where $I^{t d}$ is a dark threshold to separate the occurrence of dark intensities associated with steep wave fronts. Selection of the appropriate thresholds is not a straightforward process because it is apparent that no universal values exist, most notably in the case of the optical signal. A clear example of this would be the comparison of Fig. 3 d), e) and f), which although they exhibit a peak in the upper right quadrant, the optical threshold value appears to decrease as the environmental conditions evolved. We use visual inspection to select the threshold values that appear to provide the best detection rate on a run-by-run basis. It was found that an intensity value $I^{t}=2 / 3 * \bar{I}(\vec{x})_{\max }$ yielded good results, where $\bar{I}(\vec{x})$ is the mean of each pixel time series. The radar threshold showed less variability and was set to $\sigma_{0}^{t}=-28 \mathrm{~dB}$. Both values are fairly low and attempts to use the thresholds on each sensor independently yielded poor results, with large false detection rates.

Fig. 4 shows the performance of the joint method of detection, where a random snapshot from each sensor is overlaid with the spatial contours corresponding to each of the discrimination rules. Active breaking is well detected when compared with the visual signature, especially under conditions when the roller fronts are not so easily discernible, such as the event at $x=280 \mathrm{~m}, y=670 \mathrm{~m}$. Foam patches and steepening waves in turn are also clearly identified. The method struggles in properly detecting steepening wave fronts throughout the image, partly due to the very narrow intensity dynamic range of unbroken waves. A $I^{t d}$ value too low would be capable of detecting only the steeper waves, whereas a value too high will include all waves. This effect can be seen as a large area of unbroken waves which are identified as wave fronts near $x=200 \mathrm{~m}$. True wave fronts, however, were not identified at the far range $(x=500 \mathrm{~m}$ and beyond). However, it is considered that large steep waves scattering strongly are the most interesting feature, due to the implication for the understanding of the radar scattering mechanisms as previous research has shown that steep waves can scatter as brightly as breaking waves (e.g. Puleo et al., 2003). It was considered that the method was successful in detecting these large steep events and therefore fine tuning of the dark values was not pursued.

Next, the discrimination results are coupled with the data from RiverRad, in order to analyze the microwave scattering for each source using rigorously calibrated radar data. Results of this exercise are shown in Fig. 5, where the median of all the events at a given range (thus grazing angle $\theta_{g}$ ) are classified by source and polarization state. From the RiverRad data (center and left panels) it can be seen that there is a change in the grazing angle dependency for $\theta_{g}>3.5^{\circ}$. This a result of the antenna gain pattern affecting the calibration. While the absolute NRCS values are erroneous for $\theta_{g}>3.5^{\circ}$ (ground ranges $r<200$ $\mathrm{m})$, the relative power between sources is still useful. Breaking waves have scattering levels that are a few $\mathrm{dB}$ larger than those of steep waves and foam. The scattering from breaking waves seems to be insensitive to the polarization state and environmental conditions, whereas steep waves and foam have a slight bias 

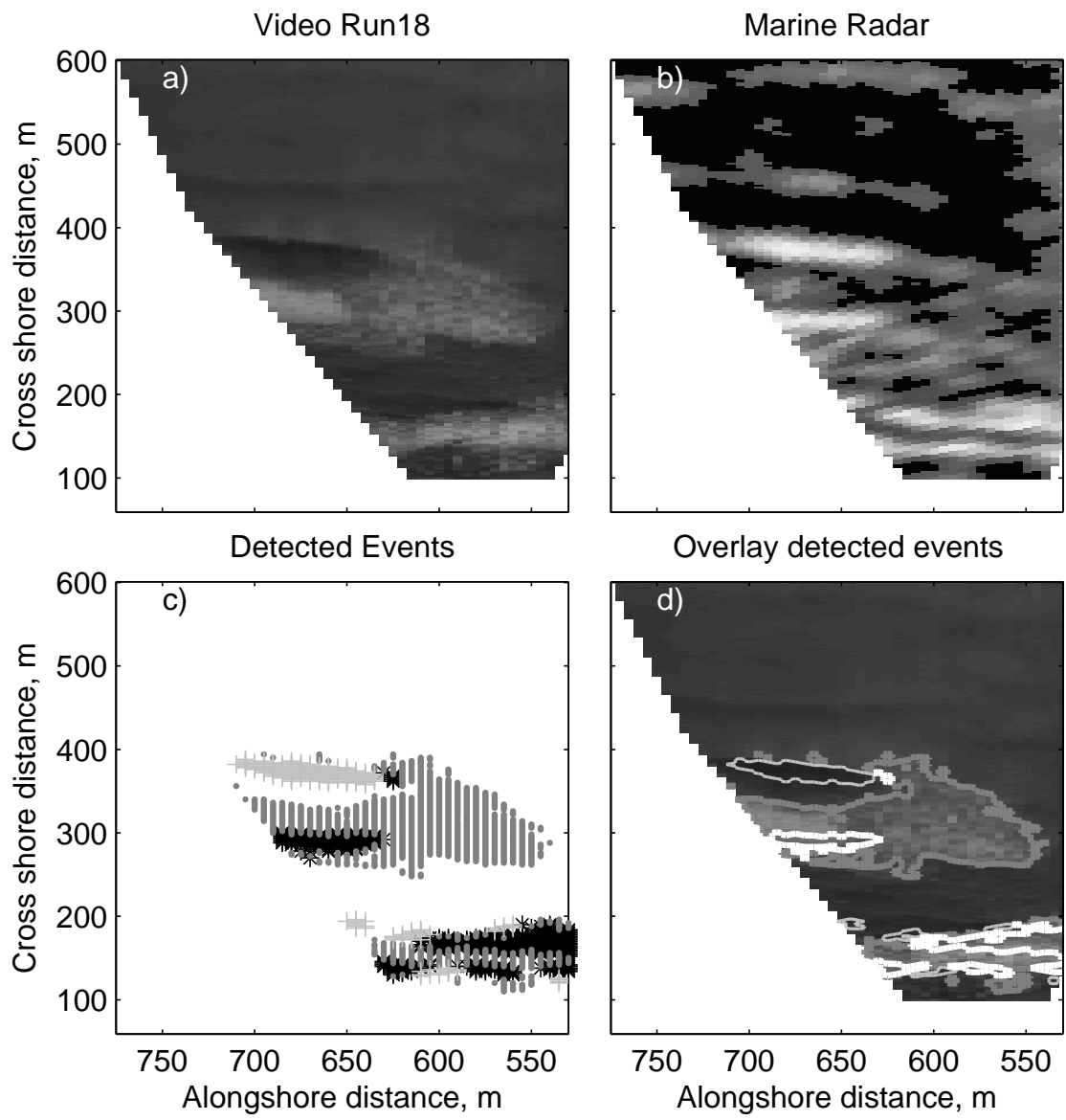

Overlay detected events

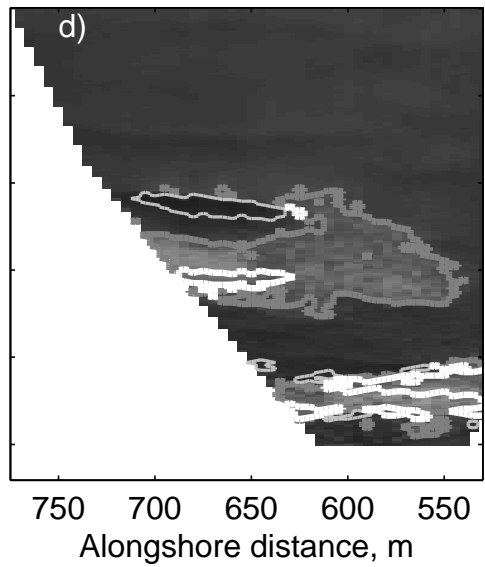

Figure 4. Combined breaking detection (Run 18). (a) Video snapshot; (b) Marine Radar snapshot; (c) Discrimination results. Black markers denote breaking; gray markers denote remnant foam; light gray markers denote steep waves. (d) Same as (c), but overlaid as contours over the video snapshot. Thin lines denote steep waves; dark gray solid lines denote foam and solid white lines denote breaking.

toward VV returns being larger than $\mathrm{HH}$ returns. Additionally, steep waves also showed a dependence on environmental conditions not shown here. Finally, steep waves and foam correlate well with the $\theta_{g}^{4}$ dependence of Bragg scattering. The scattering results from breaking waves can not be explained by existing scattering models, therefore in the following section a new model for the scattering from the wave roller is introduced.

\section{MODEL DESCRIPTION}

The wave roller is modeled as a single layer of droplets above the underlying 

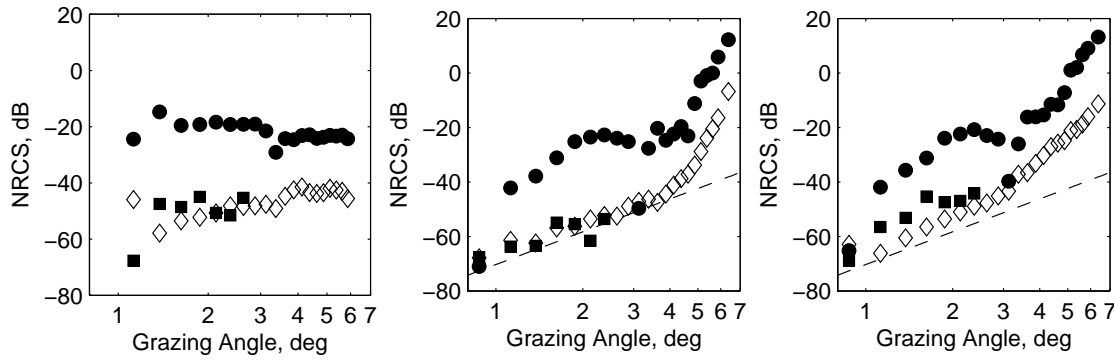

Figure 5. Grazing angle dependency of the median of the backscattered power for the ensemble of all detected events and classified by source. Breakers (circles), steep waves (squares), and foam (diamonds). Dashed line corresponds to $\theta_{g}^{4}$ trend. Marine radar (left); RiverRad HH (center) and VV (right).

wave surface. The backscatter coefficient of the layer is modeled using a first order, Dense Media Radiative Transfer (DMRT) theory. Under this approach, scattering and absorption of the incident electromagnetic fields by the particles are accounted for including coherent and incoherent multiple interactions between the scattered fields and also interactions with the media boundaries, which is the water surface in this case. Collective scattering effects are included by means of the quasi-crystalline approximation (QCA) to account for the extinction coefficient of the dense media. The method requires a few physical input parameters such as the single particle diameter $d$, total volume fraction $f_{v}$, a stickiness parameter $\tau$ to account for clustering, and the relative electric permittivity and operating microwave frequency. This approach is analogous to the modeling of active microwave sensing of snow, whose assumptions and equations are presented for instance in Tsang et al. (2007)

Model runs are compared with the NRCS obtained at low grazing angles from the present experiment and at mid grazing from two runs of the experiments described in Hesany et al. (1996), who used as similar remote sensor but much different grazing angles. While measurements suggest that the upper layers of the roller can be characterized by low volume fractions, $f_{v} \sim 20 \%$ or less (Coakley et al., 2001), data for the appropriate diameter is not available therefore we test a range of plausible diameters. We tested several $\left(d, f_{v}\right)$ combinations which showed that the model is capable of reproducing the observations well, as shown in Fig. 6 where the dots are median cross sections of individual events. The observed offset between data and model is due to treating the roller as a horizontal layer. Accounting for the roller tilt with respect to the flat ocean surface shifts the data to larger grazing angles and improves the agreement. In Fig. 6 Bragg NRCS values (thin lines) are included. These are several $\mathrm{dB}$ lower than the measured data, specially at $\mathrm{HH}$. 


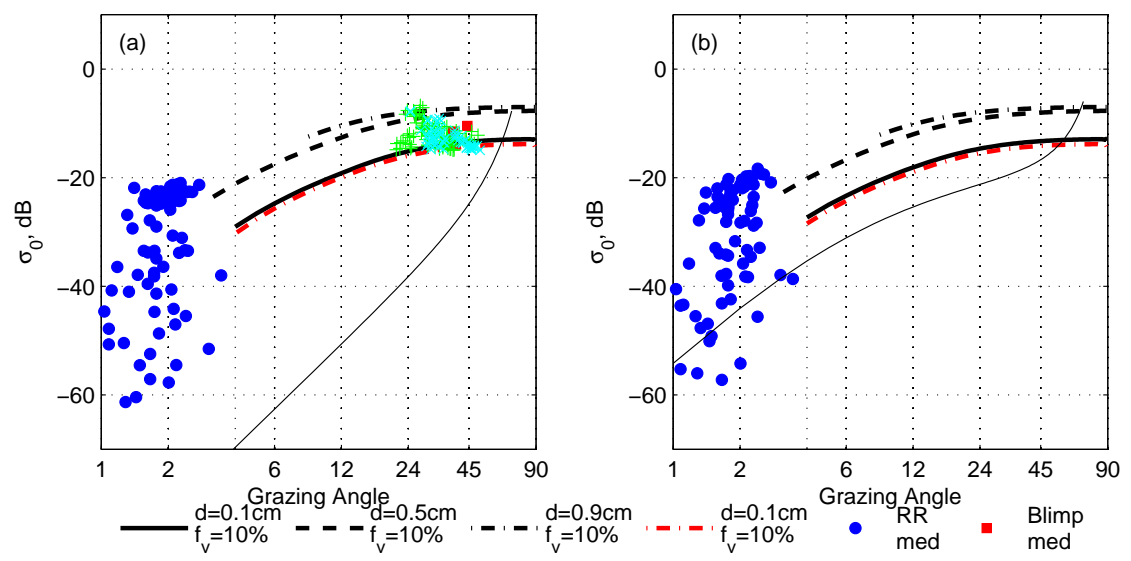

Figure 6. Model data comparison. Model runs correspond to low volume fractions $\left(f_{v}=10 \%\right)$ for three particle diameters $(d=0.1,0.5$ and $0.9 \mathrm{~cm}$ and sticky particles $(\tau=$ $0.1)$. Thin solid lines correspond to Bragg scattering. $(+)$ and $(\times)$ correspond to the data collected by Hesany et al. (1996). (a) HH; (b) VV.

\section{CONCLUSIONS}

This study comprises data analysis from a unique field experiment and modeling effort aimed at improving our understanding of the characteristics of microwave scattering from waves in the surf zone. It was found that the signal from the video sensor and the marine radar showed a consistent qualitative structure where active breaking yields large returns in both sensors. Steep waves could yield large microwave returns but low optical values, and foam typically corresponds to the opposite. Thus a joint threshold-based discrimination method was used and shown to be able to separate active breaking, steep waves and remnant foam. The discrimination results were coupled with calibrated data to perform a quantitative analysis of the microwave signal. Breaking waves show large backscattered power at both polarizations which is independent of environmental conditions and has a weak grazing dependency. Traditional scattering models from the ocean such as Bragg scattering are not capable of explaining the observations, thus a volumetric model accounting for the roller structure is introduced, yielding good agreement with the measured data.

\section{ACKNOWLEDGMENTS}

The authors wish to thank Dennis Trizna (Imaging Science Research), the staff of the Field Research Facility, John Stanley (Coastal Imaging Lab) and Gene Chatham (APL-UW) for their support during the field experiment. This work was partially supported by the Office of Naval Research under award numbers N00014-02-1-0147 and N00014-07-1-0490. 


\section{REFERENCES}

Coakley, D. B., P. M. Haldeman, D. G. Morgan, K. R. Nicolas, D. R. Penndorf, L. B. Wetzel, and C. S. Weller. 2001, May. Electromagnetic scattering from large steady breaking waves, Experiments in Fluids, 30(5), 479-487.

Gommenginger, C., N. Ward, G. Fisher, I. Robinson, and S. Boxall. 2000. Quantitative microwave backscatter measurements from the ocean surface using digital marine radar images, Journal of Atmospheric and Oceanic Technology, 17, 665-678.

Gotwols, B. L. and D. R. Thompson. 1994, 1994. Ocean microwave backscatter distributions, Journal of Geophysical Research, 99(C5), 9741-9750.

Hesany, V., W. Plant, W. Keller, and K. Hayes. 1996, May. Observed space-time structure of radar backscatter from the ocean surface, IEEE International Geoscience and Remote Sensing Symposium, 1996., Volume 3, 1733-1735.

Holman, R. and J. Stanley. 2007. The history and technical capabilities of Argus, Coastal Engineering, 54, 477-491.

Jähne, B., J. Klinke, and S. Waas. 1994. Imaging of short ocean wind waves: a critical theoretical review, J. Opt. Soc. Am. A, 11(8), 2197-2209.

Plant, W., W. Keller, and K. Hayes. 2005, June. Measurement of river surface currents with coherent microwave systems, IEEE Transactions on Geoscience and Remote Sensing, 43(6), 1242-1257.

Plant, W. J.. 1990. Bragg sea scattering of electromagnetic waves from the air/sea interface, G. L. Gernaert and W. J. Plant (Eds.), Surface Waves and Fluxes, Volume II, Chapter 11, 41-108. Kluwer Academic Publishers.

Puleo, J. A., G. Farquharson, S. J. Frasier, and K. T. Holland. 2003. Comparison of optical and radar measurements of surf and swash zone velocity fields, Journal of Geophysical Research, 108(C3), 3100.

Tsang, L., J. Pan, D. Liang, Z. Li, D. Cline, and Y. Tan. 2007, April. Modeling active microwave remote sensing of snow using Dense Media Radiative Transfer (DMRT) theory with multiple-scattering effects, IEEE Transactions on Geoscience and Remote Sensing, 45(4), 990-1004.

Wetzel, L. B.. 1990. Electromagnetic scattering from the sea at low grazing angles, G. L. Gernaert and W. J. Plant (Eds.), Surface Waves and Fluxes, Volume II, Chapter 12, 109-172. Kluwer Academic Publishers. 
KEYWORDS - ICCE 2008

SURF ZONE WAVE BREAKING IDENTIFICATION USING MARINE RADAR

Patricio Catalán, Merrick Haller,Rob Holman and William Plant Abstract Number: 552

Breaking waves

Remote sensing

Microwave radar

Marine radar 